\title{
Crucial importance of evaluating internal standards (IS) response and troubleshooting in effective LCMS method development, validation and sample analysis
}

\author{
Seongeun Julia Cho ${ }^{1}$, Stephen Vinter $^{2}$ \& Fabio Garofolo*,3 \\ ${ }^{1}$ US FDA, Silver Spring, MD, USA \\ $1 * *$ This article reflects the views of the author and should not be construed to represent FDA's views or policies. \\ ${ }^{2}$ UK MHRA, London, UK \\ ${ }^{3}$ Angelini Pharma, Rome, Italy \\ *Author for correspondence: fabiogarofolo@hotmail.com
}

First draft submitted: 21 September 2019; Accepted for publication: 27 September 2019; Published online: 22 October 2019

Internal standards (IS) are commonly used in chromatographic analytical methods to correct for variability in sample processing and analysis. To achieve this, a constant amount of IS is added to all samples - except for a double blank in a batch - including calibration standards (CALS), quality controls (QCs), and subject samples prior to the initiation of extraction procedures. Therefore, by design, the responses of IS from all samples in an analytical run are anticipated to be similar to each other. However, in some instances, we observe variability in IS responses, even among samples processed and analyzed concurrently. It is worthwhile to note that since the IS selected for a method should have similar physicochemical properties of the analyte of interest, variability of IS response in many cases may not impact the accuracy of the data, provided that IS behaves in a similar fashion as the analyte and reflects changes to the analyte that occur during sample processing and analysis. Under these conditions, for example, if ranges of IS responses for CALS, QCs and subject samples are comparable across an entire analytical run, IS variability in subject samples is unlikely to impact the accuracy of the data. In other circumstances, however, IS response variability may require additional investigations to ensure measurements of subject sample concentrations are accurate. These situations may include when the range of IS responses for subject samples is substantially wider than the range of IS responses for CALS/QCs in the same analytical run or when IS responses for subject samples are consistently lower or higher than IS responses for CALS/QCs in a run. Often, analytical laboratories rely on a pre-set acceptance rule specified in SOPs to identify study samples with unacceptable IS responses. However, handling IS irregularity solely based on a pre-set criteria and subsequent routine repeat analysis are not always effective in detecting potential problems nor provide sufficient information regarding the root causes of the variability or data accuracy.

Recently, the US FDA published a final guidance for industry, 'Evaluation of internal standard responses during chromatographic bioanalysis: questions and answers' [1]. The guidance explains when observed IS response variability may impact the accuracy of data and whether it warrants further investigation. In addition, an excerpt from the WRIB Decennial Index [2] highlights the numerous industry/regulators' discussions over the years:

"There has been much discussion between regulators and industry regarding internal standard (IS) variability, with little concrete recommendations regarding criteria. In 2011, the consensus was that when properly using a stable-labeled IS, there may not be a need for IS response acceptance criteria, as long as the stable-labeled IS and the drug of interest coelute [3]. This was reconfirmed in 2015 [4]. Discussions on this topic continued in 2014, where it was concluded that IS response evaluation is a complex topic with many scenarios to consider. A single set of criteria for IS acceptance or triggering an investigation may not be practical. A variety of approaches have been employed/discussed as appropriate acceptance criteria for IS response variation. These include numerical boundaries based on the IS variation of known samples, trend analysis and statistical methods to identify outliers. Whatever the approach taken, and whether a general set of criteria or assay-specific criteria are established, the IS response should be closely monitored; abnormal variability and outliers should be investigated [5]. Discussions in 2015 elaborated that trends and systemic differences should be 
investigated to identify their root causes and to determine effects on the accuracy of the drug/analyte concentration results in matrix. Investigations should be science-driven with clear rationale and documentation. SOPs with acceptance limits for IS response variation should be established to identify technical problems during sample processing" [4].

IS Variability was revisited in 2017 with further clarifications on this topic by US FDA. The case studies and scientific discussions among the community led to the recommendations that "During study sample analysis, thorough and consistent approaches with predefined acceptance criteria for IS responses are important to ensure accuracy of results." And "A priori criteria should consider scientific merits. Additional investigations and proper documentation may be warranted when unusual IS responses are observed" [6]. Moreover, it was decided that more training was needed to harmonize industry practice and regulatory expectations. Hence, a full day industry/regulators training session with numerous case studies and panel discussions were organized during the $12^{\text {th }}$ WRIB (2018) in Philadelphia [7]. This session, entitled 'Crucial importance of correct internal standards (IS) response troubleshooting \& interpretation in effective LCMS method development, validation and sample analysis', has been extremely successful, and it has been expanded in this special issue aimed to heighten an appropriate attention to IS responses during method development and need for critical evaluation during the sample analysis.

Articles in this issue have been inspired by the industry/regulators' interactive discussion during the $12^{\text {th }}$ WRIB and they discuss specific case studies and in-depth evaluations of the findings on IS variability. In an editorial, Stephen Vinter (MHRA; London, UK) provides an insight into inspectors' experiences and expectations of this important topic [8]. It describes the crucial role inspections play in the regulatory process and invites the reader to consider the impact IS response variations can have on bioanalytical data. Alongside this, Gustavo Mendes Lima Santos and Eduardo Fernandes (Brazilian Health Regulatory Agency [ANVISA]; Brasilia, Brazil) discuss the perspective of the ANVISA and their regulation monitoring [9]. Specifically, this article considers requirements regarding IS monitoring in CROs. Fraier et al. (Roche Innovation Center; Basel, Switzerland) demonstrates how IS response variations should trigger investigations into the root cause [10]. Through two cases, they conclude determining the cause may not always be possible, however, assay accuracy and reliability of results should be demonstrated, and re-development of an assay may be required in some situations. Similarly, Buonarati and Schoener (Intertek USA Inc, CA, USA) present examples of atypical IS responses, including biomarker assays, and conclude that close monitoring of IS response should be routine practice and unusual and substantial trends should always be evaluated [11]. Woolf (Merck \& Co, PA, USA) describes cases in his article in order to illustrate how variable IS responses cannot always be attributed to compensation of matrix effects [12]. He details that anomalous IS responses, even for stable label IS, should be investigated and the root cause should be determined where possible. Verhaeghe (Janssen Research \& Development, Beerse, Belgium) goes on to present two case studies where there was a clear response difference between study samples and CALS and QCs [13]. Verhaeghe concludes that IS acceptance criteria is useful for enabling unbiased rejection of results, however, there is no one-size-fits-all criteria and scientific judgement should be used. Similarly, van de Merbel et al. (PRA Health Sciences, Assen, The Netherlands) discuss examples of complex and extreme variation of IS responses in their article [14]. van de Merbel et al. goes on to recommend a decision-tree covering four major general areas of IS variability to assist with root cause analysis. In a Perspective by Green et al. (LGC Limited, Cambridgeshire, UK), considerations for selecting an IS in bioanalytical assays and similar challenges that Green and her team have experienced are discussed [15]. Finally, Le Blaye (French National Agency for Medicines and Health Products Safety [ANSM]; Saint-Denis, France) discusses how objective numerical criteria are useful for deciding to re-analyze individual samples, however, a visual inspection of plots of IS response could be better for monitoring trends or systematic differences [16]. This perspective concludes standard operating procedures are only capable of describing the standard response to standard situations and should not be used to justify blindly accepting data.

\section{Acknowledgments}

The guest editors would like to thank Rhiannon Finnie (Commissioning Editor, Bioanalysis) for her great enthusiasm, dedication and continuous support during the various development stages of this Special Issue on Internal Standards Variability.

\section{Disclaimer}

This article reflects the views of the author and should not be construed to represent FDA's views or policies. 


\section{Financial \& competing interests disclosure}

The authors have no relevant affiliations or financial involvement with any organization or entity with a financial interest in or financial conflict with the subject matter or materials discussed in the manuscript. This includes employment, consultancies, honoraria, stock ownership or options, expert testimony, grants or patents received or pending, or royalties.

No writing assistance was utilized in the production of this manuscript.

\section{References}

1. US Department of Health and Human Services FDA Center for Drug Evaluation and Research (CDER). Evaluation of internal standard responses during chromatographic bioanalysis: questions and answers guidance for industry (2019). www.fda.gov/media/130451/download

2. Garofolo W, Savoie N. The decennial index of the White Papers in bioanalysis: a decade of recommendations (2007-2016). Bioanalysis 9(21), 1681-1704 (2017).

3. Garofolo F, Rocci M, Dumont I et al. 2011 White Paper on recent issues in bioanalysis and regulatory findings from audits and inspections. Bioanalysis 3(18), 2081-2096 (2011).

4. Welink J, Fluhler E, Hughes N et al. 2015 White Paper on recent issues in bioanalysis: focus on new technologies and biomarkers (part 1 - small molecules by LCMS). Bioanalysis 7(22), 2913-2925 (2015).

5. Fluhler E, Hayes R, Garofolo F et al. 2014 White Paper on recent issues in bioanalysis: a full immersion in bioanalysis (part 1 - small molecules by LCMS). Bioanalysis 6(22), 3039-3049 (2014).

6. Welink J, Yang E, Hughes N et al. 2017 White Paper on recent issues in bioanalysis: aren't BMV guidance/guidelines 'scientific'? (part 1 - LCMS: small molecules, peptides and small molecule biomarkers). Bioanalysis 9(22), 1807-1825 (2017).

7. Welink J, Xu Y, Yang E et al. 2018 White Paper on recent issues in bioanalysis: 'a global bioanalytical community perspective on last decade of incurred samples reanalysis (ISR)' (part 1 - small molecule regulated bioanalysis, small molecule biomarkers, peptides \& oligonucleotide bioanalysis). Bioanalysis 10(22), 1781-1801 (2018).

8. Vinter S. Variations in internal standards: an inspector's perspective. Bioanalysis 11(18), 1649-1651 (2019).

9. Santos GML, Fernandes E. Internal standards (IS) monitoring in CROs: ANVISA perspective. Bioanalysis 11(18), 1653-1655 (2019).

10. Fraier D, Ferrari L, Heinig K, Zwanziger E. Inconsistent internal standard response in LC-MS/MS bioanalysis - an evaluation of case studies. Bioanalysis 11(18), 1657-1667 (2019).

11. Buonarati M, Schoener D. Investigations beyond SOP on internal standard response. Bioanalysis 11(18), 1669-1678 (2019).

12. Woolf E. Learning how to interpret "dangerous" internal standard behaviors. Bioanalysis 11(18), 1679-1685 (2019).

13. Verhaeghe T. Systematic IS variability and issue resolution. Bioanalysis 11(18), 1687- 1694 (2019).

14. van de Merbel NC, Koster RA, Ohnmacht C. Very complex internal standard response variation in LC-MS/MS bioanalysis: root cause analysis and impact assessment. Bioanalysis 11(18), 1695- 1702 (2019).

15. Green R, Wright M, Wheller R, Wallace G. Putting in place a decision-making process during method development based on internal standard response. Bioanalysis 11(18), 1703-1715 (2019).

16. Le Blaye O. Variations in internal standard response: some thoughts and real-life cases. Bioanalysis 11(18), 1717- 1726 (2019). 
